\title{
Nachgefragt
}

\section{Psychosoziale Interventionen bei tumorbedingter Fatigue}

?Frau Dr. Heußner, wie erkennen Sie, ob ein Krebspatient, der sich noch lange nach einer Chemotherapie antriebslos und erschöpft fühlt, an einer tumorbedingten Fatigue leidet oder etwa an einer Depression? Dr. Pia Heußner: Das ist häufig ein diagnostischer Prozess, der nicht mit einer einmaligen Exploration zu klären ist. Es gibt aber Hinweise: So ist ein schwer depressiver Patient oft so antriebsgemindert, dass er gar nicht auf die Idee kommt, irgendetwas tun zu wollen. Er sitzt auf dem Sofa und wird von seiner Familie angesprochen: „Komm, es scheint die Sonne, lass uns einen Spaziergang machen." Der Depressive sagt "Nein, ich mag nicht." Der Fatigue-Patient wird im Zweifelsfall sagen: "Oh, die Sonne scheint, ich möchte so gerne.. Und wenn er die Schuhe angezogen hat, ist er bereits so erschöpft, dass er sagt: „Ich kann nicht.“ Das heißt, der FatiguePatient ist häufig extrem änderungsmotiviert, aber er erlebt dieses "Ich kann nicht".

? Wie lässt sich herausfinden, womit einem bestimmten Fatigue-Patienten am besten geholfen ist? Heußner: Zunächst geht es um die Bestandsaufnahme: Wie ist das psychosoziale Belastungsmuster des Patienten? Ist es ausschließlich das Thema der körperlichen Erschöpfung, ist es eine psychische Anpassungsstörung oder geht es zusätzlich z.B. um finanzielle Engpässe? Erst wenn ich das Profil meines Patienten kenne, kann ich einen individuellen Maßnahmenplan erstellen. Häufig ist es sinnvoll, dass das Ausdauertraining nicht nur von Trainern begleitet wird, sondern dass eine psychoonkologische Betreuung dazu kommt. Auch fragen wir, ob Entspannungsmaßnahmen bereits bekannt sind, oder ob wir diese erst trainieren müssen. Viele Patienten haben ihre eigenen Methoden, wenden diese aber aktuell nicht an. Dann geht es eher um die Edukation. Für Patienten mit dysfunktionalen Gedankengängen sind kognitive verhaltenstherapeutische Maßnahmen angezeigt. Bei Patienten mit erheblichen Konfliktpotenzialen in der Familie geht es auch darum, an diesen zu arbeiten, um Blockaden zu lösen.
? Welche Maßnahmen sind Ihrer Erfahrung nach am erfolgreichsten?

Heußner: An erster Stelle steht der edukative Anteil, nämlich die Betroffenen, aber auch die Familienangehörigen darüber zu informieren, dass Fatigue etwas ist, das nichts mit Nicht-Wollen des Patienten zu tun hat, sondern ein definitives aktuelles Nicht-Können darstellt. Es hilft wenig, wenn die Angehörigen wohlmeinend sagen: „Nun reiß dich doch zusammen, natürlich machen wir jetzt einen Spaziergang." Und dann schleifen sie den Patienten mit und wundern sich, dass er restlos erschöpft ist und in der Folge resigniert und vielleicht aufgrund der dauernden Misserfolge tatsächlich eine Depression entwickelt.

? Das heißt, körperliches Training kann im Einzelfall auch kontraproduktiv sein?

Heußner: Absolut. Entscheidend ist, das individuell machbare Maß festzulegen. Langfristig wollen wir zwar alle Patienten dahin bringen, moderates Ausdauertraining machen zu können. Das kann aber bedeuten, dass am Anfang Spaziergänge wirklich nur zehn Minuten lang sind. Man muss also gucken, wo starte ich mit einem Patienten und was kann er ohne Frustration ganz langsam aufbauen.

? Bisherige Studien zur tumorbedingten Fatigue haben sich hauptsächlich mit Brustkrebspatientinnen befasst. Sind denn die verschiedenen Maßnahmen auch bei Patienten mit anderen Tumorentitäten wirksam?

Heußner: Ja, absolut. Traditionell sind Brustkrebspatientinnen die größte und aktivste Gruppe unter den Betroffenen. Die Patientinnen engagieren sich sehr bereitwillig in solchen Untersuchungen. Dadurch ist in der Vergangenheit extrem viel gerade an dieser Entität geforscht worden. Wir führen ein moderates Ausdauertraining aber genauso gut auch mit anderen wie Kolon-, Prostatakarzinom- oder Leukämiepatienten durch.

sierte Fragebögen, die entweder direkt auf die Fatigue abzielen, wie der Brief Fatigue Inventory (BFI) und der Multidimensional Fatigue Inventory (MFI-20), oder die Fatigue als Aspekt der Lebensqualität thematisieren (Functional Assessment of Cancer Therapy-FatigueSkala: FACT-F). Angesichts der hohen Prävalenz empfiehlt das NCI in jedem Fall ein allgemeines Screening aller Krebspatienten, das Fatigue-Symptome anhand einer von 0 bis 10 reichenden Skala erfassen soll.

\section{Wichtig ist die Abgrenzung zur Depression}

Was Diagnostik und Therapie erschwert, ist die Tat-

sache, dass Ursachen und Folgen der tumorbedingten

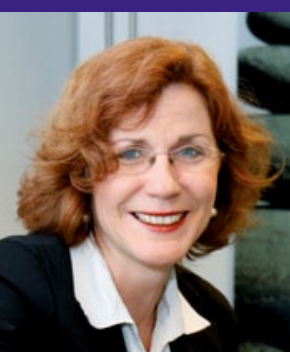

Dr. Pia Heußner

Leitung der PsychoOnkologie des Comprehensive Cancer Center der Ludwig-Maximilians-Universität München (CCC $\left.{ }^{\mathrm{LMU}}\right)$ fragt werden: Schmerz, emotionaler Stress, Schlafstörungen, Anämie und Schilddrüsenunterfunktion. Trifft keiner dieser Punkte zu, ist ein umfassenderes dige körperliche Untersuchung, eine sorgfältige Medikamentenanamnese, das Erfassen von Komorbiditäten sowie die Frage nach Ernährungsgewohnheiten und dem aktuellen körperlichen Aktivitätslevel.

Zur Diagnostik der tumorbedingten Fatigue hat die Fatigue Coalition des NCI bereits 1998 eine Checkliste erarbeitet, die allerdings bislang nicht in die ICD-10 aufgenommen wurde (Tabelle 1). Eine diagnostische Hilfestellung für die Praxis sind außerdem standardi- 